\title{
Plain Language Summaries of Clinical Trial Results: A Preliminary Study to Assess Availability of Easy-to-Understand Summaries and Approaches to Improving Public Engagement
}

\author{
Michael Penlington ${ }^{1} \cdot$ Harold Silverman ${ }^{2} \cdot$ Ajithkumar Vasudevan $^{3} \cdot$ Priya Pavithran $^{1}$
}

Accepted: 26 September 2020 / Published online: 28 October 2020

(c) The Author(s) 2020

\begin{abstract}
Background Easy-to-understand, stand-alone factual summaries of clinical trial results have the potential to improve public understanding of and engagement with pharmaceutical research. The European Clinical Trial Regulation (EU) No. 536/2014 is a major regulatory initiative that will result in a large number of such plain language summaries (PLSs) posted in the public domain. Today, however, little is known about the extent to which PLSs are written and are available to the general public. Objectives This preliminary study assessed (i) 20 top pharmaceutical companies' positions on improving transparency and commitment to disclosing trial result summaries in an easy-to-understand format and (ii) the availability of such summaries in the public domain and the ease of locating them via general web searches.

Methods The availability of PLSs in the public domain was estimated based on the number of EudraCT technical result summaries in four disease areas: chronic obstructive pulmonary disease, asthma, meningitis, and influenza. The likelihood of PLSs being easy to find through internet search engine queries by members of the public was assessed using Google.

Results All 20 sponsors had committed to improve clinical trial transparency, 17 committed to sharing PLSs with trial participants, and 14 had at least one PLS available in the public domain. A total of 99 clinical studies in these four disease areas had technical summaries posted on EudraCT between 1 January 2017 and 30 June 2020. Of these 99, 14 studies had PLSs in the public domain. A total of 12 of 14 PLSs were directly captured by search engine. However, the sponsor trial identifier or EudraCT number had to be included in the search term to locate them. Generic search terms resulted in large volumes of non-relevant results.

Conclusion Despite the progressive movement towards clinical trial transparency, easily accessible PLSs on clinical trials are currently scarce. The provision of a European mandate and framework for non-technical result summaries by Regulation (EU) 536/2014 will be a major step to bring about positive change.
\end{abstract}

\section{Plain Language Summary}

More patient and public involvement in healthcare research will help to speed the process of making new medicines. This is known by both the regulators and the healthcare industry. The healthcare industry wants to make more information on human research studies available to patients and the public. One way to help achieve this is to write simple summaries of clinical study results. Here, we use the term plain language summary (PLS) to describe them. The PLS allows people to understand human research studies more clearly. A new law will soon make it necessary to write a PLS for every clinical study done in Europe. But, today, is the PLS being used to inform the public about clinical research studies? And what is

Digital Features To view digital features for this article go to https://doi.org/10.6084/m9.figshare.12997565.

Michael Penlington

michael.c.penlington@gsk.com

GSK, Wavre, Belgium

2 GSK, Rockville, MD, USA

3 GSK, Bengaluru, India 
its potential? We found only a few researchers have already begun to write PLSs. PLSs on most studies are not available to the public. Even those PLSs on public websites are very hard to find through a Google search. To better understand the potential of PLSs we are doing more research. This research will look at what the public wants to know about these studies and how they will retrieve this information.

\section{Key Points}

The top 20 pharmaceutical companies have public positions committing to clinical trial transparency; 17 of them committed to sharing simple patient-focused summaries with trial participants, 9 indicated they would share such summaries with the public, and 14 of them had posted at least one of these in the public domain.

Relatively few completed studies (14/99) with technical summaries posted in EudraCT in four major disease areas had publicly available patient-focused summaries.

The few plain language summaries in the public domain are not easily detected by internet search engine, thereby limiting their utility as a vehicle to improve public health literacy on clinical trials.

\section{Digital Features}

To view digital features for this article go to https://doi. org/10.6084/m9.figshare.12997565.

\section{Introduction}

Patients, advocacy groups, and the general public are demanding greater transparency of information on clinical studies $[1,2]$. Sharing objective, easily accessible, unbiased research information in a timely manner complements other initiatives to build trust, partnership, and engagement throughout the clinical study process [2]. Ultimately, improvement in public health literacy and trust between the pharmaceutical industry and the public will help develop new medicines [1] and vaccines. Clearly, the industry still has some way to go before relevant information on every clinical trial becomes ubiquitously available to all. Reviews of posting requirements for technical research summaries on EudraCT and clinicaltrials.gov have revealed gaps in compliance [3-5]. Nonetheless, the clinical trial transparency movement has progressively evolved [6]. Initiatives promoting trial transparency have been announced by the FDA (the FDA Amendments Act of 2007), the European Medicines
Agency (Directive [EU] 2001/20/EC), and journal editors [7]. Posting requirements from national authorities have increased, with some now requiring disclosure of the full protocols and clinical study reports $[8,9]$.

Initiatives on disclosure of technical clinical trial material have undoubtedly increased the availability of information to those working in clinical research. However, the information they present is typically complex and not easily understood by people who do not have a scientific or medical background [10]. In addition, these postings may not be easy to find without prior knowledge of the existence of specialized hosting portals. The utility of these technical documents for patients and the general public may therefore be limited.

Consequently, there has been a push to establish policies and practices to facilitate patient and public involvement and participation in clinical research. In the European Union, Clinical Trial Regulation (Regulation [EU] No 536/2014) is a major step towards improving the transparency of clinical research, thereby potentially increasing engagement with patients and the public [11]. This regulation creates the Clinical Trials Information System (CTIS) for studies conducted within The European Union. It adds the requirement for posting easy-to-understand summaries (known as plain language summaries, patient lay summaries, simple summaries, and trial results summaries [collectively referred to as PLSs here]) of all clinical trials in addition to the currently required technical results summaries. In the US, the Public Health Service Act (2007) also requires a summary of the clinical trial and its results in non-technical, understandable language, although this is yet to be implemented due to challenges in establishing statutory standards. We expect other regulators to follow.

There is increasing evidence [12-14] that study participants want to learn about their disease, the effectiveness and safety profile of the drug they received, and whether the study will contribute towards improved public health. Shalowitz and Miller [14], for example, found that $90 \%$ of respondents wanted to receive study results. Although we know that study participants who receive a patient-focused summary value them [12], less is known about the level of interest of the general public in clinical trials. Preliminary evidence from our own unpublished data (in preparation) on vaccines and the accounts of others [15] suggest that clinical research is seen as important for the development of new medicines and vaccines. Despite growing public interest in clinical research and the pending European clinical trial 
regulation, little is known about the availability and accessibility of such documents in the public domain [2].

This preliminary study assessed (i) 20 top pharmaceutical companies' positions on improving transparency and commitment to disclosing trial result summaries in an easy-to-understand format and (ii) the availability of such summaries in the public domain and the ease of locating them via general web search.

\section{Methods}

To estimate how many of the large commercial sponsors of clinical trials are currently preparing PLSs, we identified the 20 top pharmaceutical companies by 2019 revenue [16]. Next, we searched the websites of these companies for public positions on both improving transparency and committing to writing PLSs for patients or the general public. Clinical trials sponsor websites and various other portals (e.g., trialsummaries.com [17], CISCRP.org [18]) were analyzed to identity how many of these companies had posted at least one PLS of a clinical study in the public domain.

The percentage of studies with publicly available PLSs was calculated for two areas of traditional pharma research (chronic obstructive pulmonary disease [COPD] and asthma) and two prominent areas of interest for vaccines (meningitis and influenza). To establish a baseline, the total number of technical result summaries posted on EudraCT in each of these four disease areas between 1 January 2017 and 30 June 2020 was determined. As of 31 August 2020, the number of these studies with PLSs in the public domain was determined as described above. The 'findability' of these PLSs by the public was assessed by simulating potential search patterns for clinical trial results using Google; the search engine that accounts for $87 \%$ of market share [19]. Search terms were selected based on guidance by ClinicalTrials.gov and EudraCT (e.g., [20]). Search strings contained terms such as 'clinical trial', disease (e.g., COPD, chronic obstructive pulmonary disease) and the drug name (e.g., influenza vaccine or glycopyrronium/formoterol fumarate). Sponsor trial identifier and the EudraCT trial identifier, both with and without the disease name, were also used in the search queryies. Other disease areas were also included in search terms to estimate how easy it was to identify any patient-focused summary of clinical trial results through a search engine. This assessment was not limited to PLSs of studies with technical result summaries posted on EudraCT. The search queries went from general (e.g., lay summaries of vaccine research, plain language results of vaccine research studies) to more specific search strings. Relevancy was ascertained by checking the top 50 hits for any content on patient-focused clinical trial information.

\section{Results}

\subsection{Sponsor Policies on Transparency and Sharing of Clinical Trial Results}

All 20 of the top commercial sponsors committed to improving data transparency. In total, 17 of the 20 sponsors also committed to sharing plain language information on clinical trials with trial participants and 9 indicated their intent to also share these summaries with the public. At least one PLS on any disease area had been publicly posted by 14 of the 20 sponsors.

\subsection{Plain Language Summary (PLS) Availability}

Publicly posted PLSs were identified for 14 of 99 (14\%) completed trials on the four selected diseases with technical result summaries posted on EudraCT (Table 1). We could not find a PLS for the remaining 85 studies.

\subsection{Retrieving Selected PLSs by Internet Search Engine}

When used in the same search, the terms 'clinical trial' and the disease (e.g., asthma, COPD, influenza, or meningitis) returned tens of millions of hits, although none of the 14 PLSs were identified in the top 50. Combining the term 'clinical trial' with the drug name (e.g., formoterol fumarate, influenza vaccine) also failed to identify any of the 14 PLSs in the top 50. Using the sponsor's unique trial identifier alone resulted in a large variation in the number of hits depending on the complexity of the identifier (e.g., HZA113719, 205724, D5970C00002). Repeat searches produced different numbers of hits, although they were in the same order of magnitude. Most searches using the sponsor's unique trial

Table 1 The number of studies with EudraCT result postings and PLSs

\begin{tabular}{lll}
\hline & Technical summary posted & $\begin{array}{l}\text { PLS in } \\
\text { the public } \\
\text { domain }\end{array}$ \\
\hline Asthma & 59 & 05 \\
COPD & 22 & 07 \\
Influenza & 11 & 01 \\
Meningitis & 07 & 01 \\
Total & 99 & 14 \\
\hline
\end{tabular}

$C O P D$ chronic obstructive pulmonary disease, $P L S s$ plain language summaries 
Table 2 Finding plain language summaries with technical result summaries on EudraCT through Google

\begin{tabular}{|c|c|c|c|c|c|c|}
\hline & \multirow{2}{*}{$\begin{array}{l}\text { Search by "clinical } \\
\text { trial" and disease }\end{array}$} & \multirow{2}{*}{$\begin{array}{l}\text { Search by drug/ } \\
\text { vaccine name }\end{array}$} & \multicolumn{2}{|c|}{ Sponsor's trial identifier } & \multicolumn{2}{|c|}{ EudraCT identifier } \\
\hline & & & Alone & With disease & Alone & With disease \\
\hline Asthma & 0 & 0 & 01 & 05 & 04 & 04 \\
\hline COPD & 0 & 0 & 01 & 05 & 04 & 04 \\
\hline Influenza & 0 & 0 & 0 & 01 & 01 & 01 \\
\hline Meningitis & 0 & 0 & 0 & 01 & 01 & 01 \\
\hline Total & 0 & 0 & 2 & 12 & 10 & 10 \\
\hline
\end{tabular}

Trials with EudraCT technical summary postings on four major categories of diseases between 1 January 2017 and 30 June 2020

$C O P D$ chronic obstructive pulmonary disease identifier alone did not directly identify the PLS (Table 2) in the top 50 hits. A total of 12 of the 14 PLSs were identified when including the disease with the sponsor's unique trial identifier in the same search. Searching against the EudraCT number alone resulted in direct first page hits for 10 of 14 corresponding PLSs. The results remained the same when including the disease in the search string (Table 2). Four of the missing PLSs did not have the EudraCT number referenced within the document. Two of them also omitted a reference to the sponsor's unique trial identifier.

\subsection{Retrieving ANY Patient-Focused Clinical Trial Result Summaries Through Search Strategies}

When the search was extended to any patient-focused summary on individual clinical trials, a large volume of search hits was retrieved (Table 3). None of these searches yielded a clinical trial patient-focused summary within the top 50 hits. However, links were found to some sponsor websites or general discussions about legislation, transparency, and engagement with patients and the public. Adding the disease or the name of the drug or vaccine studied to the search string also did not result in direct hits. Table 3 contains searches for PLSs on meningitis trials and is illustrative of search results in other disease areas (not shown). When the disease, and the terms 'study' and 'plain language summary' were included in the search query, the hits often included a link to the site hosting the PLS. The respective PLS was the only hit when including the sponsor's unique trial identifier in the search string.

\section{Discussion}

Understandable, easy-to-read summaries of clinical research have been recognized as providing greater transparency to those interested in learning about clinical trial results [2]. As such, the PLS may become an important component in the movement to increase public awareness and subsequently stimulate engagement in clinical research. This movement is certainly gaining momentum among clinical trials sponsors. All 20 of the top pharmaceutical companies in this

Table 3 Search for any plain language summary (PLS) through Google

\begin{tabular}{|c|c|c|}
\hline Search term & Number of results & Specificity \\
\hline "Lay summaries of vaccine research" & 7.6 million & $\begin{array}{l}\text { Provides links to some company sites where information is } \\
\text { shared with patients, many links to related general discus- } \\
\text { sions }\end{array}$ \\
\hline "Plain language results of vaccine research studies" & 4.46 million & $\begin{array}{l}\text { Provides links to some company sites describing their position } \\
\text { on disclosing data to patients, and many links to related } \\
\text { general discussions }\end{array}$ \\
\hline Plain language results of meningitis vaccine research studies & 602,000 & $\begin{array}{l}\text { Many more specific results on meningitis, meningitis vaccines, } \\
\text { meningitis vaccine side effects, relevant journal articles, no } \\
\text { PLS }\end{array}$ \\
\hline $\begin{array}{l}\text { Meningitis, Haemophilus influenzae type b study result } \\
\text { "plain language summary" }\end{array}$ & 913 & $\begin{array}{l}\text { Most results were directed toward a published journal article } \\
\text { or overview (such as the Cochrane Library). However, the } \\
\text { site where the PLS of this specific study is published (e.g., } \\
\text { the sponsor's study register) was not included }\end{array}$ \\
\hline $\begin{array}{l}\text { Meningitis, Haemophilus influenzae type b Study } 112931 \\
\text { result "plain language summary" }\end{array}$ & 1 & $\begin{array}{l}\text { When the sponsor's study number was included, the website } \\
\text { containing the published PLS was the only result }\end{array}$ \\
\hline
\end{tabular}

“" were used to denote an exact search term 
exploratory investigation, and $85 \%$ of pharmaceutical companies in general [21], have made public commitments to improving transparency and patient engagement. While the commitment is clear, implementation of these company positions may be at an early stage, as sponsors face challenges such as funding and building capability [1,22]. We do not know how many PLSs have been written and distributed to patients to date, but their availability in the public domain is limited. However, the fact that 14 of the 20 companies we looked at had publicly posted one or more PLS is an encouraging signal, especially since the European Regulation (EU) No 536/2014 mandating PLSs is still not in force. Nonetheless, there is still a long way to go.

Our finding that relatively few studies have a publicly available PLS is broadly consistent with previous accounts that production of PLSs remains scant. Getz and FaridesMitchell [1] suggested that $<2 \%$ of all clinical trials completed or terminated in a 3-year period had returned a PLS to study participants. The higher percentage (14\%) of studies with PLSs reported here reflects our focus on publicly posted PLSs (rather than those directly returned to participants) for studies that had technical results postings on EudraCT. Goldacre et al. [4], reported that half of all trials were noncompliant with this European Commission requirement for posting technical summaries.

The utility of the PLS, however, goes beyond simply producing them in a timely manner and their subsequent posting on a publicly accessible website. Better public engagement predicates that they are also easy to find. And, while 12 of the 14 PLSs were indexed by Google, we failed to identify relevant hits when simulating general public search patterns. When we broadened our search for any PLS of clinical trials, regardless of disease or whether a technical summary had been posted on EudraCT, we again found the results to be disappointing. As expected, we achieved more relevant hits as the search terms became more specific. Direct hits were achieved only when using the sponsor's unique trial identifier within the search query. It therefore appears that PLSs would not be easy to find by curious members of the public attempting to learn more about specific clinical drug, vaccine or disease trials.

While our finding on the sparsity of PLSs is consistent with published accounts by others [1], it should be noted that this is a pilot study. It is based on a limited sample size. The estimation of frequency of PLSs was confined to four major disease areas with technical result summaries posted on EudraCT. We did not search the ClinicalTrials.gov website. The authors accept that there may be a favorable bias as study sponsors prepare for implementation of the European regulation (EU) No 536/2014. In the absence of comprehensive published accounts, we can only guess how the public will search for information. Even then, search hits can vary among individuals for a given query because ever-evolving search engine algorithms personalize user experience. However, this is unlikely to be beneficial if the material cannot be easily found in the first place.

\section{Conclusion}

Overall, these results illustrate that PLSs are generally not publicly available and when they are, they are not easy to retrieve through search engines. We can expect the numbers of PLSs to increase as the European Commission Regulation (EU) No 536/2014 approaches implementation. Nonetheless, the utility of this regulation will not reach full potential until PLSs containing digestible information relevant to patients and the public are easy to locate through indexing and resultranking of search engines. Considering the potential of the PLS to improve the clinical research process, and based on the outcome of this preliminary research, we are conducting a larger international investigation probing the potential of these documents to engage the public together with what the public would most like to learn from such trials.

Acknowledgements Authors thank Géraldine Drevon, Christine Vanderlinden, Julie Vandekerckhove, Geetha Subramanyam and Philibert Goulet for critically reviewing this article.

\section{Declarations}

Funding The development of this manuscript and the open access fee was funded by GlaxoSmithKline.

Conflict of interest All authors are full-time employees of and have shares in the GlaxoSmithKline group of companies.

Ethics approval Not applicable.

Consent to participate Not applicable.

Consent for publication Not applicable.

Availability of data and material Not applicable.

Code availability Coding was not used to identify study summaries either through search on web portals or through search engines.

Author contributions All authors contributed, critically reviewed drafts of the manuscript and approved the final version.

Open Access This article is licensed under a Creative Commons Attribution-NonCommercial 4.0 International License, which permits any non-commercial use, sharing, adaptation, distribution and reproduction in any medium or format, as long as you give appropriate credit to the original author(s) and the source, provide a link to the Creative Commons licence, and indicate if changes were made. The images or other third party material in this article are included in the article's Creative Commons licence, unless indicated otherwise in a credit line to the material. If material is not included in the article's Creative Commons licence and your intended use is not permitted by statutory 
regulation or exceeds the permitted use, you will need to obtain permission directly from the copyright holder. To view a copy of this licence, visit http://creativecommons.org/licenses/by-nc/4.0/.

\section{References}

1. Getz K, Farides-Mitchell J. Assessing the adoption of clinical trial results summary disclosure to patients and the public. Expert Rev Clin Pharmacol. 2019;12(7):573-8. https://doi.org/10.1080/17512 433.2019.1615441 (Accessed 24 Jun 2019).

2. Barnes A, Patrick S. Lay summaries of clinical study results: an overview. Pharm Med. 2019;33:261-8. https://doi.org/10.1007/ s40290-019-00285-0.

3. Anderson ML, Chiswell K, Peterson ED, et al. Compliance with results reporting at clinicaltrials.gov. N Engl J Med. 2015;372:1031-9.

4. Goldacre B, DeVito NJ, et al. Compliance with requirement to report results on the EU Clinical Trials Register: cohort study and web resource. BMJ. 2018;362:k3218. https://doi.org/10.1136/bmj. k3218.

5. Piller C. FDA and NIH let clinical trial sponsors keep results secret and break the law. https://www.sciencemag.org/ news/2020/01/fda-and-nih-let-clinical-trial-sponsors-keep-resul ts-secret-and-break-law?utm_campaign=news_daily_2020-0113\&et_rid $=34822472 \&$ et_cid $=3162276 \&$ utm_source $=$ Globa 1+Health+NOW+Main+List\&utm_campaign $=2 \mathrm{f} 645 \mathrm{daf} 7 \mathrm{e}$ -EMAIL_CAMPAIGN_2020_01_13_01_49\&utm_mediu $\mathrm{m}=$ email\&utm_term $=0 \_8 \mathrm{~d} 0 \mathrm{~d} 062 \mathrm{dbd}-2 \mathrm{f} 645 \mathrm{daf7e}-897391$. Accessed 17 Aug 2020.

6. Getz K. Reflections on the evolution of patient engagement in drug development. Pharm Med. 2019;33:179-85. https://doi. org/10.1007/s40290-019-00284-1.

7. Anon. Recommendations for the Conduct, Reporting, Editing, and Publication of Scholarly Work in Medical Journals. http://www. icmje.org/icmje-recommendations.pdf. Accessed 17 Aug 2020.

8. ClinicalTrials.gov PRS: Final rule (42 CFR Part 11) information. https://prsinfo.clinicaltrials.gov/. Accessed 7 Sep 2020.

9. Health Canada. Public release of clinical information regulation: guidance document. 2019. https://www.canada.ca/en/healt h-canada/services/drug-health-product-review-approval/profi le-public-release-clinical-information-guidance/document.html. Accessed 8 Sep 2020.

10. Shailes S. Plain-language Summaries of research: something for everyone. eLife. 2017;6:e25411. https://elifesciences.org/artic les/25411.

11. Baronikova S, Purvis J, et al. Commitments by the biopharmaceutical industry to clinical trial transparency: the evolving environment. BMJ Evid-Based Med. 2019;24(5):177-84.

12. CISCRP: 2019 Perceptions and insights study. https://www.ciscr p.org/services/research-services/perceptions-and-insights-study/. Accessed 4 Aug 2020.

13. EFPIA/EFCGP. Good lay summary practice. https://efgcp.eu/ documents/GoodLaySummaryPractice_PublicConsultation 19 9.pdf. Accessed 27 July 2020.

14. Shalowitz DI, Miller FG. Communicating the results of clinical research to participants: attitudes, practices, and future directions. PLoS Med. 2008;5(5):e91. https://doi.org/10.1371/journ al.pmed.0050091.

15. Anderson A, Borfitz D, Getz K. Global public attitudes about clinical research and patient experiences with clinical trials. JAMA Netw Open. 2018;1(6):e182969. https://doi.org/10.1001/jaman etworkopen.2018.2969.

16. Fierce Pharma. The top 20 pharma companies by 2019 revenue. 2020. https://www.fiercepharma.com/special-report/top-20-pharm a-companies-by-2019-revenue Accessed 20 July 2020.

17. TRS: Helping You Stay Informed. https://www.trialsummaries. com/Home/LandingPage Accessed 26 Aug 2020.

18. CISCRP: https://www.ciscrp.org/ Accessed 26 Aug 2020.

19. Price C. 17 Great Search Engines You Can Use Instead of Google. https://www.searchenginejournal.com/alternative-search-engin es/271409/\#close. Accessed 17 Aug 2020.

20. Clinical Trials: What Patients Need to Know. https://www.fda. gov/patients/clinical-trials-what-patients-need-know. Accessed 26 Aug 2020.

21. Grom T. Driving patient engagement. PharmaVOICE March 2020. https://www.pharmavoice.com/article/2020-03-patient-engag ement/ Accessed 17 Aug 2020.

22. Getz, K.A. Measuring adoption and value of patient engagement Appl Clin Trial. 2017;26(2):13-14. https://www.appliedclinical trialsonline.com/view/measuring-adoption-and-value-patientengagement. 